\title{
The Impact of Faculty Status and Gender on Employee Well-being in Academic Libraries
}

\section{Quinn Galbraith, Leanna Fry, and Melissa Garrison}

This study measures job satisfaction, personal fulfillment, work/life balance, and stress levels of male and female librarians. Researchers surveyed 719 librarians at ARL institutions that either offer faculty status and tenure or offer neither. Females at libraries offering faculty status indicated poor work/life balance and high levels of stress compared to male colleagues and female librarians without faculty status; however, their reported job satisfaction was similar. Possible implications of the results are discussed.

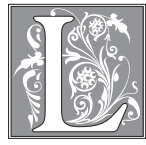

ibrarianship has long been a field dominated by females. The 2013-2014 ARL Salary Survey reported that 62.2 percent of academic librarians were female. ${ }^{1}$ However, female librarians have still encountered inequality issues experienced by women in other fields. In 1999-2000, males made up only 36.5 percent of academic librarians, yet 51.4 percent of academic library directors were male. ${ }^{2}$ Over the years, these numbers have changed. While males still account for 37.8 percent of academic librarians, in 2013-2014 the ratio of male directors went down to 40.7 percent, a ratio certainly more representative of the actual proportion of male and female librarians. ${ }^{3}$ However, the 2013-2014 ARL Salary Survey also reported discrepancies in the salaries of female librarians compared to male. ${ }^{4}$ These inconsistencies suggest research is needed in other areas where female and male librarians may differ in their work experiences. Using a survey instrument, this study asked academic librarians to rate several values including their levels of job satisfaction, stress, and work/life balance. Although male and female librarians characterized themselves as experiencing comparable job satisfaction, female librarians at tenure-track institutions identified themselves as working longer hours, feeling more stress at work, and experiencing poorer work/life balance than their male counterparts.

\section{Literature Review}

Stress in the workplace has been well documented in library literature but has not focused on gender differences. Bunge identified librarians' top three stressors as patrons,

Quinn Galbraith is Sociology and Family Life Librarian, Leanna Fry is Advanced Writing Librarian, and Melissa Garrison is Research Assistant in Harold B. Lee Library at Brigham Young University; e-mail: quinn_galbraith@byu.edu, leanna_balci@byu.edu, melissa_garrison@byu.net. We appreciate the assistance of Sean Miner, Research Assistant at Brigham Young University. (C) 2016 Quinn Galbraith, Leanna Fry, and Melissa Garrison, Attribution-NonCommercial (http://creativecommons.org/licenses/by-nc/3.0/) CC BY-NC. 
workload, and supervisors and management..$^{5}$ Shupe and Pung found that stressors included "difficulties related to employees' roles, including role ambiguity, role overload, and role conflict" and also cited Saddiq and Burke's study reportedly suggesting that "librarians experience higher levels of stress than firefighters, teachers, or police officers." ${ }^{6}$ Bronstein identified the changing roles of librarians, particularly in terms of technology, as a source of stress, and in a case study by Farler and Broady-Preston, the "need to 'control' noise levels, modify student behaviour and balance the needs of different user groups are cited as stressors." ${ }^{7}$ Larrivee focused on new librarians, narrowing sources of stress to a new work culture, self-expectations, job relocation, and crossover stress. ${ }^{8}$ Multiple articles also explored librarians and burnout. ${ }^{9}$ Maslach and Jackson defined burnout as a "syndrome of emotional exhaustion and cynicism that occurs frequently among individuals who do 'people-work' of some kind."10 Becker identified a relationship between burnout and library instruction, and Affleck found that a majority of instruction librarians reported high levels of burnout, many due to role conflict. ${ }^{11}$ Harwell found high levels of burnout among business librarians, and Nelson discovered a similar trend among law librarians. ${ }^{12}$ Holcomb, Sheesley, and Harwell each identified strategies for addressing burnout, including self-reflection, professional collaborations, and even job transfers. ${ }^{13}$ Other literature suggested approaches librarians can take to manage and relieve stress, including Mastel and Innes's discussion of "mindful librarianship."14 However, while these articles identify many sources of stress unique to librarians, they fail to acknowledge the impact gender may have on librarian stress levels.

Literature outside the field of librarianship offers more insight into the relationship between gender and stress. Specifically, many studies have examined the challenges faced by gender minorities in an occupation and may therefore offer significant insight into the female-dominated occupation of librarianship. In a study involving female accountants and male nurses, Evans and Steptoe found that both of these gender minorities faced increased work hassles compared to their majority coworkers. ${ }^{15}$ The stress from these work hassles manifested itself in increased sick absences in men and increased cases of anxiety among women. ${ }^{16}$ Evans and Steptoe concluded that the minority gender in a profession had a more stressful/difficult work experience. A study by Simpson, however, identified several mechanisms men use to counter the negative effects of being a minority in the workplace such as those discussed by Evans and Steptoe. For instance, men in her study believed that "their minority status as men gave them career advantages."17 Additionally, they perceived themselves as having "greater authority than their female counterparts." ${ }^{18}$ Simpson concluded that "it was the minority group (men) that was active in creating a distance from the dominant group (women) and who claimed a higher status in the process." ${ }^{19}$ Simpson also found that "men may well benefit from preferential treatment and from exposure to roles and situations that are challenging and developmental," ${ }^{20}$ all as a result of their minority status within a female-dominated occupation. While these studies focus on other occupations, their conclusions can apply to a female-dominated occupation such as librarianship. Our study aims to examine the differences in work-experience for male and female librarians, with literature on gender minorities in other occupations informing our discussion and conclusions.

The concept of work/life balance is underrepresented in library literature. Spires mentions work/life balance in his discussion of librarian stress when he suggests that tenure-track academic librarians must find a balance among job duties, home life, and tenure requirements. ${ }^{21}$ However, literature outside librarianship may offer more insight into gender differences in work/life balance. Other fields such as human resources have explored the concept of work/life balance more thoroughly. For example, in a survey 
of IBM employees, Hill et al. discovered that "half of the employees had difficulty with work/life balance," yet "gender was not significantly correlated to work/life balance." 22

Other literature has explored gender and work/life balance specifically in terms of the academy as a whole. In exploring gender issues for teaching faculty, Ward and Wolf-Wendel focus on the difficulties faced by mothers in academic positions. Ward and Wolf-Wendel discuss many sources of stress for faculty mothers, including the "never-ending" nature of the academic workload, guilt for "spending too little time" on both family relationships and academic work, "the ambiguities of tenure expectations" and "not having enough time in the day." 23 Several articles as recently as 2014 have discussed motherhood as well, pointing out that graduate school and tenure pursuits often overlap with typical childbearing years for women. ${ }^{24}$ While discussions of motherhood are obviously not applicable to all female faculty, they may offer insight into some of the challenges facing female librarians in regard to work/life balance.

In one of the few articles focusing on academic fathers, Reddick et al. found that fathers often experience many of the same conflicts between work and family as female academics, including the pressures of tenure and not having enough time to devote to either work or family responsibilities. ${ }^{25}$ These conflicts were especially pronounced among men striving to be "active fathers," sharing the burden for parenthood equally with a partner. ${ }^{26}$ However, participants in the Reddick et al. study also admitted that biological and societal expectations for parenthood likely put greater strain on female academics. ${ }^{27}$ In a study focusing on parenthood and librarianship, Graves et al. found that female librarians were "significantly more likely than their male colleagues to postpone having children" and were more likely to perceive the negative impacts children can have on a career. ${ }^{28}$ However, the same study also found that both genders believed policies supporting work/life balance should be equally available to both male and female librarians. ${ }^{29}$ While discussions of work/life balance in librarianship and academia more broadly have generally focused on mothers, the efforts of fathers in academic positions to be a part of their children's lives and the resulting conflict that parenting creates with work/life balance should not be discounted.

Many of the faculty mothers in Ward and Wolf-Wendel's study mentioned that the flexibility inherent in their teaching-faculty positions allowed them to balance both work and family. ${ }^{30}$ However, while teaching-faculty may enjoy this flexibility, Graves et al. point out that academic librarians often do not "[enjoy] the freedom of passage granted to teaching faculty" considering that "many librarians [keep] a standard forty hour work week" and are busy "running the daily activities of libraries." ${ }^{11}$ Regardless of parental status, presumably the lack of flexibility afforded by librarianship should be considered in examining the work/life balance of both male and female librarians.

\section{Methodology}

A survey was developed, based on experience in the area of organizational behavior, asking librarians to rate indicators of employee well-being on a Likert scale (see appendix A). Initial questions asked respondents to provide limited demographic information, including rank, time at current institution, hours worked per week (librarians were instructed to leave this question blank if not a full-time employee), and gender. Where applicable, respondents were asked whether or not they had achieved tenure. They were then asked to assess various indicators of employee well-being, as measured by work/life balance, job satisfaction, stress at work, and personal fulfillment. These assessments were reported on a scale of $1-7$, with 1 being low and 7 being high.

The survey was distributed via e-mail to deans of 110 ARL libraries with the instruction that only professional librarians should take the survey. Twenty-five of these libraries responded. Of the twenty-five ARL libraries that responded, fifteen granted 
librarians faculty status and tenure, eight did not grant librarians faculty status or tenure, and two granted librarians faculty status but not tenure. For the purposes of this survey and discussion, faculty status for academic librarians is defined as granting the same privileges and rights that are granted to the teaching faculty within a university. "At some institutions, faculty status refers to academic rank (e.g., Librarian I, II or III) and the same rights and privileges of teaching faculty, whereas at others it represents the availability of tenure. Tenure, one aspect of faculty status, is continuous appointment or a commitment by an institution to provide permanent employment where one can only be terminated for adequate cause." ${ }^{32}$ These rights and privileges often include, but are not guaranteed or limited to, "corresponding entitlement to rank, promotion, tenure, compensation, leaves, and research funds." ${ }^{33}$ It should be noted that some institutions had different names for "faculty status" and "tenure." In these cases, the institution was given liberty to decide if their policies were the equivalent of faculty status and/or tenure. The institutional review board at each university, as well as the authors' affiliated institution, gave approval for the study.

Each library was sent a survey link individual to that university so that responses could be compared across institutions if necessary. However, the survey questions analyzed in this article were identical for each institution. In all, 846 librarians responded. The response rate is unknown, as it is unclear how many librarians received the survey. Of the responses collected, 527 were from libraries offering faculty status and tenure (faculty institutions), 254 from libraries offering neither (nonfaculty institutions), and sixty-five from libraries offering faculty status without tenure.

An overwhelming majority of ARL libraries either offer faculty status and tenure or offer neither. ${ }^{34}$ Responses from the two libraries granting faculty status but not tenure were compared to determine if the relatively small sample could be considered representative of any larger group. Two sample t-tests confirmed that a considerable number of responses showed statistically significant variation between the two schools in question. This, combined with the smaller size of the sample, led to the exclusion of these two libraries from further analysis, comparison, and discussion in this article.

For the remaining two groups of libraries, individual responses were excluded from analysis if the respondent did not indicate gender. For faculty institutions, responses were excluded from analysis if tenure status was not indicated. Under these conditions, 62 responses were not counted, leaving a total of 719 valid responses.

With these exclusions, 249 responses remained from nonfaculty institutions. Of these, $185(74 \%)$ respondents were female and $64(26 \%)$ respondents were male. From the faculty institutions, 470 responses remained. Of these, $319(68 \%)$ were female and 151 $(32 \%)$ were male. Out of the faculty respondents, $299(64 \%)$ had been granted tenure. Of the librarians granted tenure, 103 (34\%) were male and $196(66 \%)$ were female. Of the remaining 171 tenure-track respondents, 123 (72\%) were female and 48 were male (28\%). At 196, tenured females made up the largest group of respondents. Overall, 504 females were surveyed and 215 males. It should also be noted that 68 percent of the males in this group had achieved tenure, compared to 61 percent of females.

Responses to questions were initially analyzed using a two-tailed two-sample t-test. Equal variance was assumed. T-test results comparing genders within institution-type and tenure-status for work/life balance, stress, personal fulfillment, and job satisfaction can be found in table 1 . Results comparing genders across institution-type and tenurestatus for these same variables can be found in table 2. Responses were also analyzed using a multiple linear regression to further explore the many independent variables that may impact stress, work/life balance, personal fulfillment, and job satisfaction. A separate model was created for each dependent variable that included responses from faculty institutions only to allow the inclusion of tenure as an independent variable. 


\section{TABLE 1}

T-Test Results by Institution Type and Tenure

\begin{tabular}{|c|c|c|c|c|c|c|c|c|c|c|c|c|c|}
\hline Group & $\mathbf{n}$ & Mean & SD & df & $\mathbf{T}$ & $\mathbf{p}$ & Group & $\mathbf{n}$ & Mean & SD & df & $\mathbf{T}$ & p \\
\hline \multicolumn{7}{|c|}{ Work/Life Balance } & \multicolumn{7}{|c|}{ Personal Fulfillment } \\
\hline $\begin{array}{l}\text { Tenured } \\
\text { Male } \\
\text { Female }\end{array}$ & $\begin{array}{l}103 \\
196\end{array}$ & $\begin{array}{l}4.90 \\
4.40\end{array}$ & $\begin{array}{l}1.54 \\
1.64\end{array}$ & $\begin{array}{l}102 \\
195\end{array}$ & 2.715 & $0.0078 * * *$ & $\begin{array}{l}\text { Tenured } \\
\text { Male } \\
\text { Female }\end{array}$ & $\begin{array}{l}103 \\
196\end{array}$ & $\begin{array}{l}5.26 \\
5.33\end{array}$ & $\begin{array}{l}1.57 \\
1.32\end{array}$ & $\begin{array}{l}102 \\
195\end{array}$ & 0.376 & 0.7079 \\
\hline $\begin{array}{l}\text { Tenure Track } \\
\text { Male } \\
\text { Female }\end{array}$ & $\begin{array}{c}48 \\
123\end{array}$ & $\begin{array}{l}4.38 \\
4.18\end{array}$ & $\begin{array}{l}1.64 \\
1.57\end{array}$ & $\begin{array}{c}47 \\
122\end{array}$ & 0.734 & 0.4664 & $\begin{array}{l}\text { Tenure Track } \\
\text { Male } \\
\text { Female }\end{array}$ & $\begin{array}{c}48 \\
123\end{array}$ & $\begin{array}{l}5.08 \\
5.14\end{array}$ & $\begin{array}{l}1.32 \\
1.33\end{array}$ & $\begin{array}{c}47 \\
122\end{array}$ & 0.240 & 0.8116 \\
\hline $\begin{array}{l}\text { Faculty } \\
\text { Male } \\
\text { Female }\end{array}$ & $\begin{array}{l}151 \\
319 \\
\end{array}$ & $\begin{array}{l}4.73 \\
4.31\end{array}$ & $\begin{array}{l}1.57 \\
1.61 \\
\end{array}$ & $\begin{array}{l}150 \\
318 \\
\end{array}$ & 2.755 & $0.0066^{* * *}$ & $\begin{array}{l}\text { Faculty } \\
\text { Male } \\
\text { Female }\end{array}$ & $\begin{array}{l}151 \\
319 \\
\end{array}$ & $\begin{array}{l}5.21 \\
5.25\end{array}$ & $\begin{array}{l}1.51 \\
1.33 \\
\end{array}$ & $\begin{array}{l}150 \\
318 \\
\end{array}$ & 0.372 & 0.7102 \\
\hline $\begin{array}{l}\text { Nonfaculty } \\
\text { Male } \\
\text { Female }\end{array}$ & $\begin{array}{c}64 \\
185\end{array}$ & $\begin{array}{l}4.73 \\
4.82\end{array}$ & $\begin{array}{l}1.72 \\
1.48\end{array}$ & $\begin{array}{c}63 \\
184\end{array}$ & 0.447 & 0.6563 & $\begin{array}{l}\text { Nonfaculty } \\
\text { Male } \\
\text { Female }\end{array}$ & $\begin{array}{c}64 \\
185\end{array}$ & $\begin{array}{l}5.06 \\
5.39\end{array}$ & $\begin{array}{l}1.57 \\
1.34\end{array}$ & $\begin{array}{c}63 \\
184\end{array}$ & 1.652 & 0.1035 \\
\hline \multicolumn{7}{|l|}{ Level of Stress } & \multicolumn{7}{|l|}{ Job Satisfaction } \\
\hline $\begin{array}{l}\text { Tenured } \\
\text { Male } \\
\text { Female }\end{array}$ & $\begin{array}{l}103 \\
196\end{array}$ & $\begin{array}{l}4.46 \\
5.04\end{array}$ & $\begin{array}{l}1.48 \\
1.33\end{array}$ & $\begin{array}{l}102 \\
195\end{array}$ & 3.521 & $6.50 \mathrm{E}-4 * * * *$ & $\begin{array}{l}\text { Tenured } \\
\text { Male } \\
\text { Female }\end{array}$ & $\begin{array}{l}103 \\
196\end{array}$ & $\begin{array}{l}5.14 \\
5.05\end{array}$ & $\begin{array}{l}1.57 \\
1.39\end{array}$ & $\begin{array}{l}102 \\
195\end{array}$ & 0.482 & 0.6311 \\
\hline $\begin{array}{l}\text { Tenure Track } \\
\text { Male } \\
\text { Female }\end{array}$ & $\begin{array}{c}48 \\
123 \\
\end{array}$ & $\begin{array}{l}4.75 \\
5.17\end{array}$ & $\begin{array}{l}1.33 \\
1.46\end{array}$ & $\begin{array}{c}47 \\
122 \\
\end{array}$ & 1.714 & $0.0932 *$ & $\begin{array}{l}\text { Tenure Track } \\
\text { Male } \\
\text { Female }\end{array}$ & $\begin{array}{c}48 \\
123 \\
\end{array}$ & $\begin{array}{l}5.06 \\
4.84\end{array}$ & $\begin{array}{l}1.54 \\
1.58\end{array}$ & $\begin{array}{c}47 \\
122 \\
\end{array}$ & 0.873 & 0.3871 \\
\hline $\begin{array}{l}\text { Faculty } \\
\text { Male } \\
\text { Female }\end{array}$ & $\begin{array}{l}151 \\
319\end{array}$ & $\begin{array}{l}4.55 \\
5.09\end{array}$ & $\begin{array}{l}1.48 \\
1.38\end{array}$ & $\begin{array}{l}150 \\
318\end{array}$ & 3.907 & $1.40 \mathrm{E}-4 * * * *$ & $\begin{array}{l}\text { Faculty } \\
\text { Male } \\
\text { Female }\end{array}$ & $\begin{array}{l}151 \\
319\end{array}$ & $\begin{array}{l}5.11 \\
4.97\end{array}$ & $\begin{array}{l}1.56 \\
1.46\end{array}$ & $\begin{array}{l}150 \\
318\end{array}$ & 0.971 & 0.3333 \\
\hline $\begin{array}{l}\text { Nonfaculty } \\
\text { Male } \\
\text { Female }\end{array}$ & $\begin{array}{c}64 \\
185\end{array}$ & $\begin{array}{l}4.62 \\
4.73\end{array}$ & $\begin{array}{l}1.58 \\
1.48\end{array}$ & $\begin{array}{c}63 \\
184\end{array}$ & 0.543 & 0.5892 & $\begin{array}{l}\text { Nonfaculty } \\
\text { Male } \\
\text { Female }\end{array}$ & $\begin{array}{c}64 \\
185\end{array}$ & $\begin{array}{l}5.06 \\
5.02\end{array}$ & $\begin{array}{l}1.59 \\
1.47\end{array}$ & $\begin{array}{c}63 \\
184\end{array}$ & 0.204 & 0.8391 \\
\hline
\end{tabular}




\begin{tabular}{|c|c|c|c|c|c|c|c|c|c|c|c|c|c|}
\hline \multicolumn{14}{|c|}{$\begin{array}{c}\text { TABLE } 2 \\
\text { T-Test Results by Gender }\end{array}$} \\
\hline Group & $\mathrm{n}$ & Mean & SD & df & $\mathbf{T}$ & $\mathbf{p}$ & Group & $\mathrm{n}$ & Mean & SD & df & $T$ & p \\
\hline \multicolumn{7}{|c|}{ Work/Life Balance } & \multicolumn{7}{|c|}{ Personal Fulfillment } \\
\hline $\begin{array}{l}\text { Male } \\
\text { Female }\end{array}$ & $\begin{array}{l}215 \\
504\end{array}$ & $\begin{array}{l}4.73 \\
4.50\end{array}$ & $\begin{array}{l}1.61 \\
1.58\end{array}$ & $\begin{array}{l}214 \\
503\end{array}$ & 1.852 & $0.0654 *$ & $\begin{array}{l}\text { Male } \\
\text { Female }\end{array}$ & $\begin{array}{l}215 \\
504\end{array}$ & $\begin{array}{l}5.16 \\
5.30\end{array}$ & $\begin{array}{l}1.38 \\
1.37\end{array}$ & $\begin{array}{l}214 \\
503\end{array}$ & 1.256 & 0.21057479 \\
\hline $\begin{array}{l}\text { Male } \\
\text { Tenured } \\
\text { Tenure Track }\end{array}$ & $\begin{array}{c}103 \\
48\end{array}$ & $\begin{array}{l}4.90 \\
4.38\end{array}$ & $\begin{array}{l}1.54 \\
1.64\end{array}$ & $\begin{array}{c}102 \\
47\end{array}$ & 1.988 & $0.0528 *$ & $\begin{array}{l}\text { Male } \\
\text { Tenured } \\
\text { Tenure Track }\end{array}$ & $\begin{array}{c}103 \\
48\end{array}$ & $\begin{array}{l}5.26 \\
5.08\end{array}$ & $\begin{array}{l}1.57 \\
1.32 \\
\end{array}$ & $\begin{array}{l}102 \\
47\end{array}$ & 0.679 & 0.5003 \\
\hline $\begin{array}{l}\text { Female } \\
\text { Tenured } \\
\text { Tenure Track }\end{array}$ & $\begin{array}{l}196 \\
123\end{array}$ & $\begin{array}{l}4.40 \\
4.18\end{array}$ & $\begin{array}{l}1.64 \\
1.57\end{array}$ & $\begin{array}{l}195 \\
122\end{array}$ & 1.217 & 0.2258 & $\begin{array}{l}\text { Female } \\
\text { Tenured } \\
\text { Tenure Track }\end{array}$ & $\begin{array}{l}196 \\
123\end{array}$ & $\begin{array}{l}5.33 \\
5.14\end{array}$ & $\begin{array}{l}1.32 \\
1.33\end{array}$ & $\begin{array}{l}195 \\
122\end{array}$ & 1.238 & 0.2180 \\
\hline $\begin{array}{l}\text { Male } \\
\text { Faculty } \\
\text { Nonfaculty }\end{array}$ & $\begin{array}{c}151 \\
64\end{array}$ & $\begin{array}{l}4.73 \\
4.73\end{array}$ & $\begin{array}{l}1.57 \\
1.72\end{array}$ & $\begin{array}{c}150 \\
63\end{array}$ & 0.004 & 0.997 & $\begin{array}{l}\text { Male } \\
\text { Faculty } \\
\text { Nonfaculty }\end{array}$ & $\begin{array}{c}151 \\
64\end{array}$ & $\begin{array}{l}5.21 \\
5.06\end{array}$ & $\begin{array}{l}1.51 \\
1.57\end{array}$ & $\begin{array}{c}150 \\
63\end{array}$ & 0.628 & 0.5321 \\
\hline $\begin{array}{l}\text { Male } \\
\text { Female }\end{array}$ & $\begin{array}{l}215 \\
504\end{array}$ & $\begin{array}{l}4.57 \\
4.96\end{array}$ & $\begin{array}{l}1.51 \\
1.42\end{array}$ & $\begin{array}{l}214 \\
503\end{array}$ & 3.342 & $9.80 \mathrm{E}-4 * * * *$ & $\begin{array}{l}\text { Male } \\
\text { Female }\end{array}$ & $\begin{array}{l}215 \\
504\end{array}$ & $\begin{array}{l}5.10 \\
4.99\end{array}$ & $\begin{array}{l}1.56 \\
1.56\end{array}$ & $\begin{array}{l}214 \\
503\end{array}$ & 0.917 & 0.3600 \\
\hline $\begin{array}{l}\text { Male } \\
\text { Tenured } \\
\text { Tenure Track }\end{array}$ & $\begin{array}{c}103 \\
48\end{array}$ & $\begin{array}{l}4.46 \\
4.75\end{array}$ & $\begin{array}{l}1.48 \\
1.33\end{array}$ & $\begin{array}{c}102 \\
47\end{array}$ & 1.149 & 0.2567 & $\begin{array}{l}\text { Male } \\
\text { Tenured } \\
\text { Tenure Track }\end{array}$ & $\begin{array}{c}103 \\
48\end{array}$ & $\begin{array}{l}5.14 \\
5.06\end{array}$ & $\begin{array}{l}1.57 \\
1.54\end{array}$ & $\begin{array}{c}102 \\
47\end{array}$ & 0.273 & 0.7864 \\
\hline $\begin{array}{l}\text { Female } \\
\text { Tenured } \\
\text { Tenure Track }\end{array}$ & $\begin{array}{l}196 \\
123\end{array}$ & $\begin{array}{l}5.04 \\
5.17\end{array}$ & $\begin{array}{l}1.33 \\
1.46\end{array}$ & $\begin{array}{l}195 \\
122\end{array}$ & 0.852 & 0.3958 & $\begin{array}{l}\text { Female } \\
\text { Tenured } \\
\text { Tenure Track }\end{array}$ & $\begin{array}{l}196 \\
123\end{array}$ & $\begin{array}{l}5.05 \\
4.84\end{array}$ & $\begin{array}{l}1.39 \\
1.58\end{array}$ & $\begin{array}{l}195 \\
122\end{array}$ & 1.274 & 0.2052 \\
\hline $\begin{array}{l}\text { Male } \\
\text { Faculty } \\
\text { Nonfaculty }\end{array}$ & $\begin{array}{c}151 \\
64\end{array}$ & $\begin{array}{l}4.55 \\
4.62\end{array}$ & $\begin{array}{l}1.48 \\
1.58\end{array}$ & $\begin{array}{c}150 \\
63\end{array}$ & 0.314 & 0.7549 & $\begin{array}{l}\text { Male } \\
\text { Faculty } \\
\text { Nonfaculty }\end{array}$ & $\begin{array}{c}151 \\
64\end{array}$ & $\begin{array}{l}5.11 \\
5.06\end{array}$ & $\begin{array}{l}1.56 \\
1.59\end{array}$ & $\begin{array}{c}150 \\
63\end{array}$ & 0.223 & 0.8243 \\
\hline $\begin{array}{l}\text { Female } \\
\text { Faculty } \\
\text { Nonfaculty }\end{array}$ & $\begin{array}{l}319 \\
185\end{array}$ & $\begin{array}{l}5.09 \\
4.73\end{array}$ & $\begin{array}{l}1.38 \\
1.48\end{array}$ & $\begin{array}{l}318 \\
184\end{array}$ & 2.723 & $0.0071 * * *$ & $\begin{array}{l}\text { Female } \\
\text { Faculty } \\
\text { Nonfaculty }\end{array}$ & $\begin{array}{l}319 \\
185\end{array}$ & $\begin{array}{l}4.97 \\
5.02\end{array}$ & $\begin{array}{l}1.46 \\
1.47\end{array}$ & $\begin{array}{l}318 \\
184\end{array}$ & 0.377 & 0.7068 \\
\hline
\end{tabular}


Models were narrowed down to exclude any insignificant variables. This reduction was implemented to separate very weak explanatory variables from stronger ones. Degrees of freedom were not consistent for each test, as blank responses in any variable being tested were excluded from analysis.

\section{Results}

\section{Work/Life Balance}

When analyzed using t-tests (see tables 1 and 2), responses for work/life balance showed a significant difference between males and females overall $(p=0.0654)$. Additional gender differences appeared when broken into groups based on institution type and tenure status. At faculty institutions, males reported statistically significantly higher work/life balance than females $(p=0.0066)$. When broken down farther, tenured males reported statistically significantly higher work/life balance than tenured females $(p=0.0078)$, while no statistically significant difference was found between tenure-track males and females. In addition, tenure-track males reported statistically significantly lower work/ life balance than tenured males $(p=0.0528)$. At nonfaculty institutions, no statistically significant differences were found between male and female work/life balance. While there was no statistically significant difference between males at each type of library, females at nonfaculty institutions reported significantly higher work/life balance than females at faculty institutions $(p=3.0 \mathrm{E}-4)$.

While t-test results did not reveal a statistically significant difference in work/life balance between males and females, regression analysis indicated that gender was a statistically significant variable in relation to other variables tested (see table 3). Regression analysis did not reveal statistically significant differences between faculty and nonfaculty librarians. However, the interaction between gender and type of institution

\begin{tabular}{|c|c|c|c|c|}
\hline \multicolumn{5}{|c|}{$\begin{array}{c}\text { TABLE } 3 \\
\text { Work/Life Balance }\end{array}$} \\
\hline & Coefficient & $\begin{array}{c}\text { Standard } \\
\text { Error }\end{array}$ & $\mathbf{T}$ & $\mathbf{P}$ \\
\hline Intercept & 8.85 & 0.395 & 22.41 & $<2 \mathrm{E}-16^{* * * *}$ \\
\hline Female & -0.29 & 0.117 & -2.46 & $0.0142 * *$ \\
\hline Years Worked at Library & 0.01 & 0.0054 & 1.68 & $0.0942 *$ \\
\hline Hours per Week & -0.09 & 0.0076 & -11.83 & $<2 \mathrm{E}-16 * * * *$ \\
\hline Interaction (Female and Nonfaculty) & 0.15 & 0.062 & 2.48 & $0.0136^{* *}$ \\
\hline Degrees of Freedom $=687$ & \multicolumn{4}{|c|}{ Multiple R-Squared $=.1928$} \\
\hline \multicolumn{5}{|c|}{ Work/Life Balance (Faculty Libraries Only) } \\
\hline & Coefficient & $\begin{array}{c}\text { Standard } \\
\text { Error }\end{array}$ & $\mathbf{T}$ & $\mathbf{P}$ \\
\hline Intercept & 9.57 & 0.509 & 18.80 & $<2 \mathrm{E}-16 * * * *$ \\
\hline Female & -0.33 & 0.141 & -2.33 & $0.0203^{* *}$ \\
\hline Without Tenure & -0.31 & 0.136 & -2.28 & $0.0228 * *$ \\
\hline Hours Per Week & -0.09 & 0.0094 & -9.82 & $<2 \mathrm{E}-16^{* * * *}$ \\
\hline \multicolumn{5}{|c|}{ Degrees of Freedom $=449 \quad$ Multiple R-Squared $=.1974$} \\
\hline$* p<0.1 \quad * * p<0.05 \quad * * * p<0.01 \quad * *$ & .001 & & & \\
\hline
\end{tabular}


was statistically significant $(p=0.0136)$, meaning the difference in work/life balance between males and females at nonfaculty schools was smaller than the gender difference at faculty schools. Average hours worked per week and the number of years a librarian has worked at his or her current institution were also found to have a statistically significant impact on work/life balance. Librarians working longer hours had poorer work/life balance ( $p<2 \mathrm{E}-16)$. The longer a librarian had been employed at his or her current institution, the higher the reported work/life balance ( $p=.0942)$.

In the model focusing on faculty libraries, gender was found to be statistically significant $(p=0.0203)$. Tenure status was also statistically significant, with librarians working toward tenure reporting poorer work/life balance than those who had already achieved tenure $(p=.0228)$. However, the interaction between gender and tenure was not statistically significant. Hours worked per week was also found to be statistically significant $(p<2 \mathrm{E}-16)$.

\begin{tabular}{|c|c|c|c|c|}
\hline \multicolumn{5}{|c|}{$\begin{array}{c}\text { TABLE } 4 \\
\text { Stress }\end{array}$} \\
\hline & Coefficient & $\begin{array}{c}\text { Standard } \\
\text { Error }\end{array}$ & $\mathbf{T}$ & $\mathbf{P}$ \\
\hline Intercept & 1.86 & 0.386 & 4.82 & $1.75 \mathrm{E}-6 * * * *$ \\
\hline Female & 0.41 & 0.116 & 3.53 & $4.50 \mathrm{E}-4 * * * *$ \\
\hline Hours Per Week & 0.053 & 0.0075 & 7.00 & $6.03 \mathrm{E}-12 * * * *$ \\
\hline Interaction (Female and Nonfaculty) & -0.11 & 0.062 & -1.79 & $0.0747 *$ \\
\hline \multicolumn{5}{|c|}{ Multiple R-Squared $=.08824$} \\
\hline \multicolumn{5}{|c|}{ Stress (Faculty Libraries Only) } \\
\hline & Coefficient & $\begin{array}{c}\text { Standard } \\
\text { Error }\end{array}$ & $\mathbf{T}$ & $\mathbf{P}$ \\
\hline Intercept & 1.49 & 0.493 & 3.02 & $0.00263 * * *$ \\
\hline Female & 0.49 & 1.493 & 3.61 & $3.35 \mathrm{E}-4 * * * *$ \\
\hline Without Tenure & 0.17 & 2.493 & 1.29 & 0.197 \\
\hline Hours Per Week & 0.05 & 3.493 & 5.79 & $1.30 \mathrm{E}-8 * * * *$ \\
\hline \multicolumn{5}{|c|}{ Degrees of Freedom $=454 \quad$ Multiple R-Squared $=.1021$} \\
\hline$* p<0.1 \quad * * p<0.05 \quad * * * p<0.01 \quad * * * *$ & $p<0.001$ & & & \\
\hline
\end{tabular}

\section{Stress}

When asked to assess their level of stress at work, a t-test (see tables 1 and 2) revealed that females overall reported a statistically significantly higher level of stress than their male colleagues ( $p=9.80 \mathrm{E}-4)$. At faculty institutions, females reported statistically significantly more stress than their male colleagues both overall ( $p=1.40 \mathrm{E}-4)$ and when limited only to librarians with tenure $(p=6.50 \mathrm{E}-4)$. There was also a statistically significant difference in the level of stress reported by tenure-track males and tenure-track females ( $p=0.0932)$. Males and females at nonfaculty institutions showed no statistically significant difference in reported stress levels. However, females at faculty institutions reported statistically significantly higher levels of stress than females at nonfaculty institutions $(p=0.007)$.

The multiple-linear regression for stress (see table 4 ) revealed that gender had a statistically significant association with level of stress $(p=4.50 \mathrm{E}-4)$. The interaction between 
gender and type of institution was also found to be statistically significant, although at a lower confidence level ( $p=0.0747)$. Still, the difference between male and female librarians at faculty schools was found to be greater than the difference at nonfaculty schools. Hours worked per week was found to be statistically significant ( $p=6.03 \mathrm{E}-12)$. A separate regression for faculty librarians only revealed that gender and hours per week had a statistically significant association with stress ( $p=3.35 \mathrm{E}-4 ; p=1.30 \mathrm{E}-8)$. Both females and librarians working more average hours per week had significantly higher stress than other librarians. Tenure status was not found to significantly impact level of stress.

\section{Hours Worked}

Average hours worked per week was analyzed as a dependent variable to determine if gender influenced hours worked. A t-test analysis of these responses appears in table 5. A statistically significant difference in number of hours worked appeared between females at the two different types of institutions. Females at faculty institutions reported working an average of 45.59 hours per week, while females at nonfaculty institutions worked an average of 43.59 hours $(p=0.0014)$. In addition, a statistically significant dif-

\begin{tabular}{|c|c|c|c|c|c|c|}
\hline \multicolumn{7}{|c|}{$\begin{array}{c}\text { TABLE } 5 \\
\text { Hours Worked per Week }\end{array}$} \\
\hline Group & $\mathbf{n}$ & Mean & SD & df & $\mathbf{T}$ & $\mathbf{p}$ \\
\hline $\begin{array}{l}\text { Male } \\
\text { Female }\end{array}$ & $\begin{array}{l}209 \\
488\end{array}$ & $\begin{array}{l}44.76 \\
44.86\end{array}$ & $\begin{array}{l}6.78 \\
6.68\end{array}$ & $\begin{array}{l}208 \\
487\end{array}$ & 0.171 & 0.864 \\
\hline $\begin{array}{l}\text { Tenured } \\
\text { Male } \\
\text { Female }\end{array}$ & $\begin{array}{l}100 \\
188\end{array}$ & $\begin{array}{l}44.63 \\
46.11\end{array}$ & $\begin{array}{l}5.48 \\
6.79\end{array}$ & $\begin{array}{c}99 \\
187\end{array}$ & 1.895 & $0.0610^{*}$ \\
\hline $\begin{array}{l}\text { Tenure Track } \\
\text { Male } \\
\text { Female }\end{array}$ & $\begin{array}{c}47 \\
120\end{array}$ & $\begin{array}{l}46.01 \\
44.78\end{array}$ & $\begin{array}{l}6.56 \\
7.48\end{array}$ & $\begin{array}{c}46 \\
119\end{array}$ & 0.996 & 0.3248 \\
\hline $\begin{array}{l}\text { Faculty } \\
\text { Male } \\
\text { Female }\end{array}$ & $\begin{array}{l}147 \\
308 \\
\end{array}$ & $\begin{array}{l}45.07 \\
45.59\end{array}$ & $\begin{array}{l}5.86 \\
7.08 \\
\end{array}$ & $\begin{array}{l}146 \\
307 \\
\end{array}$ & 0.777 & 0.4384 \\
\hline $\begin{array}{l}\text { Nonfaculty } \\
\text { Male } \\
\text { Female } \\
\end{array}$ & $\begin{array}{c}62 \\
180 \\
\end{array}$ & $\begin{array}{l}44.02 \\
43.59\end{array}$ & $\begin{array}{l}8.58 \\
5.73\end{array}$ & $\begin{array}{c}61 \\
179 \\
\end{array}$ & 0.445 & 0.6576 \\
\hline $\begin{array}{l}\text { Male } \\
\text { Tenured } \\
\text { Tenure Track }\end{array}$ & $\begin{array}{c}100 \\
47\end{array}$ & $\begin{array}{l}44.63 \\
46.01 \\
\end{array}$ & $\begin{array}{l}5.48 \\
6.56 \\
\end{array}$ & $\begin{array}{l}99 \\
46\end{array}$ & 1.351 & 0.1836 \\
\hline $\begin{array}{l}\text { Female } \\
\text { Tenured } \\
\text { Tenure Track }\end{array}$ & $\begin{array}{l}188 \\
120\end{array}$ & $\begin{array}{l}46.11 \\
44.78\end{array}$ & $\begin{array}{l}6.79 \\
7.48\end{array}$ & $\begin{array}{l}187 \\
119\end{array}$ & 0.989 & 0.3248 \\
\hline $\begin{array}{l}\text { Male } \\
\text { Faculty } \\
\text { Non-Faculty }\end{array}$ & $\begin{array}{c}147 \\
62 \\
\end{array}$ & $\begin{array}{l}45.07 \\
44.02 \\
\end{array}$ & $\begin{array}{l}5.86 \\
8.58 \\
\end{array}$ & $\begin{array}{c}146 \\
61 \\
\end{array}$ & 1.027 & 0.3087 \\
\hline $\begin{array}{l}\text { Female } \\
\text { Faculty } \\
\text { Non-Faculty }\end{array}$ & $\begin{array}{l}308 \\
180\end{array}$ & $\begin{array}{l}45.59 \\
43.59\end{array}$ & $\begin{array}{l}7.08 \\
5.73\end{array}$ & $\begin{array}{l}307 \\
179\end{array}$ & 3.246 & $0.0014 * * *$ \\
\hline
\end{tabular}


ference was found between tenured males and tenured females at faculty institutions, with tenured females working more hours on average than tenured males $(p=0.0610)$.

\begin{tabular}{|l|c|c|c|c|}
\hline \multicolumn{5}{|c|}{ TABLE 6 } \\
& Hours Worked per Week \\
\hline & Coefficient & $\begin{array}{c}\text { Standard } \\
\text { Error }\end{array}$ & T & P \\
\hline Intercept & 43.94 & 0.999 & 43.98 & $<2 \mathrm{E}-16 * * * *$ \\
\hline Female & 0.81 & 0.584 & 1.39 & 0.164 \\
\hline Interaction (Female and Nonfaculty) & -1.01 & 0.308 & -3.30 & $0.00102 * * *$ \\
\hline Degrees of Freedom $=\mathbf{6 9 9}$ & \multicolumn{5}{|c|}{ Multiple R-Squared $=\mathbf{. 0 1 5 9}$} \\
\hline$* p<0.1 \quad * * p<0.05 * * * p<0.01 \quad * * * * p<0.001$ \\
\hline
\end{tabular}

When analyzed using a multiple-linear regression (see table 6), hours worked per week was not significantly associated with gender. However, the interaction between gender and type of institution was found to be significant, with females from nonfaculty schools working less than other groups $(p=0.00102)$.

\section{Personal Fulfillment}

Responses to the question assessing personal fulfillment showed no statistically significant differences along gender lines when analyzed using t-tests (see tables 1 and 2). Across the board, all librarians, no matter their gender or university, seemed relatively happy with their positions. All averages fell above a 5, indicating librarians tend to find their jobs personally fulfilling.

\begin{tabular}{|c|c|c|c|c|}
\hline \multicolumn{5}{|c|}{$\begin{array}{c}\text { TABLE } 7 \\
\text { Personal Fulfillment }\end{array}$} \\
\hline & Coefficient & $\begin{array}{l}\text { Standard } \\
\text { Error }\end{array}$ & $\mathbf{T}$ & $\mathbf{P}$ \\
\hline Intercept & 5.11 & 0.081 & 63.17 & $<2 \mathrm{E}-16^{* * * *}$ \\
\hline Years Worked at Library & 0.01 & 0.0053 & 2.45 & $0.0147 * *$ \\
\hline \multicolumn{2}{|l|}{ Degrees of Freedom $=714$} & \multicolumn{3}{|c|}{ Multiple R-Squared $=\mathbf{0 . 0 0 8 3}$} \\
\hline \multicolumn{5}{|c|}{ Personal Fulfillment (Faculty Libraries Only) } \\
\hline & Coefficient & $\begin{array}{c}\text { Standard } \\
\text { Error }\end{array}$ & $\mathbf{T}$ & $\mathbf{P}$ \\
\hline Intercept & 5.05 & 0.101 & 50.14 & $<2 \mathrm{E}-16^{* * * *}$ \\
\hline Years Worked at Library & 0.02 & 0.0065 & 2.39 & $0.0171 * *$ \\
\hline \multicolumn{2}{|l|}{ Degrees of Freedom $=467$} & \multicolumn{3}{|c|}{ Multiple R-Squared = 0.01001} \\
\hline$* p<0.1 \quad * * p<0.05 \quad * * * p<0.01$ & $* * * * p<0.001$ & & & \\
\hline
\end{tabular}

Similar results are seen in the multiple-linear regression (see table 7). There was no statistical significance found between gender and personal fulfillment. The only variable significantly related to personal fulfillment in this model was number of years worked in the library. The longer a librarian had worked at his or her current institution, the higher the librarian's personal fulfillment was likely to be $(p=0.0147)$. Analysis of only 
faculty institutions had the same result, with years worked in the library being the only variable significantly associated with personal fulfillment $(p=0.0171)$. No statistical significance appeared along gender or tenure lines.

\section{Job Satisfaction}

Responses to the question assessing job satisfaction were similar to personal fulfillment. T-tests did not reveal any statistically significant differences in gender in these responses (see tables 1 and 2). Both males and females reported relatively high job satisfaction, with the average for males at 5.1 and the average for females at 5.0. When broken down by institution type, the average for some groups fell slightly below a $5.0-$ with the lowest average being tenure-track females at 4.84 .

\begin{tabular}{|c|c|c|c|c|}
\hline \multicolumn{5}{|c|}{$\begin{array}{c}\text { TABLE } 8 \\
\text { Job Satisfaction }\end{array}$} \\
\hline & Coefficient & $\begin{array}{c}\text { Standard } \\
\text { Error }\end{array}$ & $T$ & $\mathbf{P}$ \\
\hline Intercept & 5.55 & 0.360 & 15.41 & $<2 \mathrm{E}-16^{* * * * *}$ \\
\hline Years Worked at Library & 0.01 & 0.0056 & 2.06 & $0.0402 * *$ \\
\hline Hours per Week & -0.01 & 0.0078 & -1.88 & $0.0608 *$ \\
\hline \multicolumn{2}{|l|}{ Degrees of Freedom $=694$} & \multicolumn{3}{|c|}{ Multiple R-Squared = 0.01092} \\
\hline \multicolumn{5}{|c|}{ Job Satisfaction (Faculty Libraries Only) } \\
\hline & Coefficient & $\begin{array}{c}\text { Standard } \\
\text { Error }\end{array}$ & $\mathbf{T}$ & $\mathbf{P}$ \\
\hline Intercept & 4.82 & 0.107 & 44.84 & $<2 \mathrm{E}-16^{* * * *}$ \\
\hline Years Worked at Library & 0.02 & 0.0070 & 2.35 & $0.0191 * *$ \\
\hline \multicolumn{2}{|l|}{ Degrees of Freedom $=466$} & \multicolumn{3}{|c|}{ Multiple R-Squared = 0.01174} \\
\hline \multicolumn{5}{|c|}{$* p<0.1 \quad * * p<0.05 \quad * * * p<0.01 \quad * * * * p<0.001$} \\
\hline
\end{tabular}

Analysis using a multiple-linear regression also did not identify any statistically significant differences in gender regarding job satisfaction (see table 8). However, years worked at current position and average hours worked per week were both identified as having a statistically significant impact on job satisfaction. A higher number of hours worked per week resulted in lower job satisfaction $(p=0.0608)$, while a higher number of years worked at current position resulted in higher job satisfaction $(p=0.0402)$. Analysis of only faculty schools revealed that gender and tenure did not have a statistically significant impact on job satisfaction. Number of years worked at current library was the only variable found to have a statistically significant relationship to job satisfaction at faculty institutions $(p=0.0191)$.

\section{Discussion}

Data from this study indicate that differences exist between male and female employee well-being in the field of librarianship. Despite the sometimes common perception that females naturally experience more stress than males, the results of this current study indicate that female librarians at nonfaculty institutions had closer levels of stress and work/life balance to their male colleagues than faculty females did to faculty males. Thus, gender differences in stress and work/life balance were found to be more pro- 
nounced at faculty institutions. This discrepancy seems to suggest that female librarians in faculty positions have different experiences from their male counterparts that lead to notable differences in employee well-being. Female librarians in nonfaculty institutions, however, appear to have a different experience than their female faculty counterparts that aligned their employee well-being closer to that of males. The data do not explicitly indicate what obstacles female faculty librarians may face that lead to these discrepancies. Using the data from this current study in combination with previously published research, however, may suggest reasons for these inconsistencies.

Although respondents to this current survey were not asked to identify themselves as parents, conclusions regarding the challenges faced by teaching faculty mothers may apply to mothers in faculty librarian positions as well, helping to explain the high levels of stress and work/life imbalance faced by the female faculty librarians in this survey. Ward found that the majority of female faculty interviewed were expected to take on more responsibility for not only parenting, but also housekeeping than the male parent, a factor that may add stress and work/life balance challenges for female librarians regardless of whether or not they have children. ${ }^{35}$ In addition, the overlapping of tenure with family and child-bearing concerns for women as mentioned in the literature review may contribute to female librarians' stress and lack of work/life balance, especially for those in faculty positions seeking tenure. ${ }^{36}$ Based on the literature, the tensions between family and career responsibilities seem to be a source of pressure that could particularly impact the stress and work/life balance experienced by female librarians working at faculty institutions.

The results of our survey provide support for the findings of Graves et al., as librarians surveyed from all groups reported working, on average, well above 40 hours per week. This result supports the assertion made by Graves et al. that librarians may lack the flexibility teaching faculty enjoy. ${ }^{37}$ While this conclusion does not offer direct insight into gender issues, it is an important consideration when discussing work/life balance and stress-especially considering that our data indicate hours worked per week strongly impacted librarian work/life balance and stress. While t-tests did not indicate a significant difference in hours worked between men and women, a significant difference was seen when comparing females at faculty institutions to females at nonfaculty institutions. Regression analysis indicated that females at nonfaculty institutions were likely to work less on average than other demographic groups. Our data indicate that working more hours per week on average increases stress and negatively affects work/life balance-a finding which, combined with the results indicating nonfaculty females work less than faculty females, may help explain the discrepancies between female employee well-being at faculty and nonfaculty institutions.

The lack of statistically significant differences between male and female job satisfaction and personal fulfillment should also be noted. Neither regression nor t-test analysis suggested an association between gender and job satisfaction or personal fulfillment. Rather, years worked at current position and number of hours worked were identified as factors related to these areas of employee well-being. The lack of gender differences in these areas, despite discrepancies in male and female stress and work/life balance, may be related to a long-standing trend in job satisfaction among females. Female workers have historically reported high levels of job satisfaction. Andrew E. Clark's seminal article on gender and job satisfaction noted that "women's higher job satisfaction does not reflect that their jobs are unobservedly better than men's, but rather that, perhaps because their jobs have been so much worse in the past, they have lower expectations." 38 The fact that female and male librarians report similar job satisfaction is supported by Clark's assertion that " $t]$ he gender differential disappears for younger workers, higher-educated workers, those in professional or 
managerial positions, those whose mothers had a professional job, and those working at male-dominated workplaces." ${ }^{39}$ Additionally, Ward found that, "despite whatever frustrations they feel about the struggles of balancing children and work and the pressure of tenure," faculty mothers were able to "find joy in both their professional and personal roles." ${ }^{40}$ Many faculty members explained that they loved both their children and their jobs, with some expressing a desire to set positive examples for their children by showing them that their parents love their jobs. ${ }^{41}$ While this current survey finds that females in faculty librarian positions face substantial stress while struggling to maintain balance between work and life, they also report levels of satisfaction and fulfillment comparable to their male colleagues. As mentioned before, this survey did not ask questions related to parental status, so it is unknown to what extent motherhood may have impacted the data.

The survey results point to a positive work experience for male librarians, as demonstrated by males' lower levels of stress and higher work/life balance, despite the fact that male librarians are a minority in the field. These results are supported by Simpson's conclusion that men in minority fields are able to effectively navigate their occupation, using mechanisms to counter the challenges caused by their minority status and have a work experience superior to the majority-females..$^{42}$ According to Simpson's conclusions, then, the men in this study may have shown lower levels of stress at work because of, rather than despite, their minority status. In addition, several other factors may help explain male librarians' positive work experience. Male academic librarians may consider themselves less of a minority because of their place in academia, a male-dominated field. This double-layered majority/minority situation could certainly complicate the work experience for both male and female librarians since each could be considered both a majority and a minority.

\section{Limitations and Considerations for Future Research}

As mentioned previously, this study has several limitations. While results derived from $\mathrm{t}$-tests provided an initial indication of the possible associations between gender, faculty status, and employee well-being, the likelihood of error when performing a large number of t-tests may weaken these results. Analysis based on multiple-linear regression was performed to supplement the t-tests and provide more reliable insight into the relationships between variables. As such, results supported solely by t-tests must be viewed with caution. In addition, while the causes of the gender differences revealed by this survey can be speculated on, further research is needed to reveal the actual causes of gender differences in academic librarianship. The survey did not ask respondents about specific sources of stress or challenges to work/life balance. In addition, the survey did not inquire into the family situations of the librarians, including marital or parental status. With the recent attention that literature has given to parenthood in academia, it is reasonable to assume parenthood could be a key factor in understanding gender differences in employee well-being among academic librarians, and further research should be done to ascertain if this is the case. Where our study showed hours worked per week as a strong predictor of employee well-being, a study focusing specifically on factors influencing the number of hours worked per week by librarians may be beneficial.

\section{Conclusion}

The intersection between work life and home life has important implications for librarians. McCoy et al., in a study on the well-being of male and female faculty members, found female faculty "reported lower job satisfaction, higher intent to leave, lower emotional health, and marginally lower physical health than men." ${ }^{43}$ They also concluded 
that both male and female faculty "were happier the more support they perceived for balancing work and home life." ${ }^{44}$ Hill et al. suggested that, when discussing work/life interaction, a "metaphor of harmony instead of balance" 45 may be more appropriate. While academic librarians face different pressures than teaching faculty face, it still appears that support for harmonizing work with outside life could have positive effects for both male and female academic librarians. An improvement in work/life balance could potentially decrease work-related stress and result in happier and more productive employees. Myers Spencer recommended taking individual action to find work/life harmony, including using vacation time, learning relaxation techniques, and maintaining a social network outside of work. ${ }^{46}$ Spires also suggested that librarians organize themselves and set goals to relieve stress. ${ }^{47}$ Most literature, however, argues that librarians' stress and work/life balance is more effectively addressed at the organizational level than the individual level. ${ }^{48}$ Several articles offered suggestions for libraries seeking to improve employee well-being, such as providing mentoring relationships, offering professional development opportunities, and "managing job demands and job rewards." ${ }^{49}$ Hill et al. suggested that flexibility may be the key to finding that work/life harmony. They defined "workplace flexibility as 'the ability of workers to make choices influencing when, where, and for how long they engage in work-related tasks" ${ }^{\prime \prime 50}$ and found that "[e]mployees with perceived flexibility in the timing and location of work were able to work longer hours than those without perceived flexibility before experiencing a difficulty in balancing their work and family life." ${ }^{51}$ Organizational efforts to support workplace flexibility and employee well-being may be the most significant means through which libraries can help ease the level of stress and work/life imbalance experienced by librarians.

\section{Notes}

1. ARL Annual Salary Survey 2013-2014, eds. Martha Kyrillidou and Shaneka Morris (Washington, D.C.: Association of Research Libraries, 2014), 12.

2. ARL Annual Salary Survey 1999-2000, eds. Martha Kyrillidou and Michael O'Connor (Washington, D.C.: Association of Research Libraries, 2000), 48.

3. ARL Annual Salary Survey 2013-2014, 16.

4. Ibid., 12.

5. Charles Bunge, "Stress in the Library," Library Journal 112, no. 15 (1987).

6. Ellen I. Shupe and Stephanie K. Pung, "Understanding the Changing Role of Academic Librarians from a Psychological Perspective: A Literature Review," Journal of Academic Librarianship 37, no. 5 (2011): 410.

7. Jenny Bronstein, "The Role and Work Perceptions of Academic Reference Librarians: A Qualitative Inquiry," portal: Libraries \& The Academy 11, no. 3 (2011); Liz Farler and Judith BroadyPreston, "Workplace Stress in Libraries: A Case Study," Aslib Proceedings 64, no. 3 (2012): 236.

8. Anne Larrivee, "Exploring the Stressors of New Librarians," Public Services Quarterly 10, no. 1 (2014).

9. Mary Ann Affleck, "Burnout among Bibliographic Instruction Librarians," Library \& Information Science Research 18, no. 2 (1996); Tim Baird and Zehra M. Baird, "How to Enjoy Being an MLS: A Master of Library Stress," available online at www.liscareer.com/baird_stress.htm [accessed 23 June 2014]; K.A. Becker, "The Characteristics of Bibliographic Instruction in Relation to the Causes and Symptoms of Burnout," Rq 32, no. 3 (1993); Kevin Harwell, "Burnout Strategies for Librarians," Journal of Business \& Finance Librarianship 13, no. 3 (2008); Kevin Harwell, "Burnout and Job Engagement Among Business Librarians," Library Leadership \& Management 27, no.1/2 (2013); Deborah F. Sheesley, "Burnout and the Academic Teaching Librarian: An Examination of the Problem and Suggested Solutions," Journal of Academic Librarianship 27, no. 6 (2001).

10. Christina Maslach and Susan E. Jackson, "The Measurement of Experienced Burnout," Journal of Occupational Behaviour 2, no. 2 (1981): 1.

11. Becker, "The Characteristics of Bibliographic Instruction"; Affleck, "Burnout among Bibliographic Instruction Librarians."

12. Harwell, "Burnout and Job Engagement among Business Librarians"; Veneese C. Nelson, 
"Burnout: A Reality for Librarians?" Law Library Journal 79, no. 2 (1987).

13. Jean M. Holcomb, “Battling Burnout," Law Library Journal 99, no. 3 (2007): 673; Sheesley, "Burnout and the Academic Teaching Librarian," 449; Harwell, "Burnout Strategies for Librarians," 388.

14. Raquel J. Gabriel, "Dealing with Stress," Law Library Journal 104, no. 3 (2012); Anna Shelton, "Take Action: Managing Workplace Stress," Alki 28, no. 3 (2012); Roxanne Myers Spencer, "Taking Care of Yourself: Stress and the Librarian," Community \& Junior College Libraries 19, no. 1/2 (2013); Todd Spires, "The Busy Librarian: Prioritizing Tenure and Dealing with Stress for Academic Library Professionals," Illinois Libraries 86, no. 4 (2007); Kristen Mastel and Genevieve Innes, "Insights and Practical Tips on Practicing Mindful Librarianship to Manage Stress," LIBRES: Library E Information Science Research Electronic Journal 23, no. 1 (2013).

15. Olga Evans and Andrew Steptoe, "The Contribution of Gender-role Orientation, Work Factors and Home Stressors to Psychological Well-Being and Sickness Absence in Male- and Female-Dominated Occupational Groups," Social Science E Medicine 54 (2002): 486.

16. Ibid., 487.

17. Ruth Simpson, "Masculinity at Work: The Experiences of Men in Female Dominated Occupations," Work, Employment, and Society 18, no. 2 (2004): 356.

18. Ibid., 357.

19. Ibid., 363.

20. Ibid.

21. Spires, "The Busy Librarian."

22. E. Jeffrey Hill et al., "Finding an Extra Day a Week: The Positive Influence of Perceived Job Flexibility on Work and Family Life Balance," Family Relations 50, no. 1 (2001): 53-54.

23. Kelly Ward and Lisa Wolf-Wendel, Academic Motherhood: How Faculty Manage Work and Family (Piscataway: Rutgers University Press, 2012), 52, 55.

24. Audrey Williams June, "Fear of Stigma Lingers About Use of Family-Friendly Policies," Chronicle of Higher Education, available online at http://chronicle.com/article/Fear-of-StigmaLingers-About/146965 [accessed 25 June 2014]; Kate Bahn, “When Grad School Eats Up Your 'Good Years,'" Vitae News, available online at https://chroniclevitae.com/news/525-when-gradschool-eats-up-your-good-years [accessed 25 June 2014].

25. Richard J. Reddick, et al., "Academic Fathers Pursuing Tenure: A Qualitative Study of WorkFamily Conflict, Coping Strategies, and Departmental Culture," Psychology of Men E Masculinity 13, no. 1 (Jan. 2012): 5.

26. Ibid., 6-7.

27. Ibid., 6 .

28. Stephanie J. Graves, Jian Anna Xiong, and Ji-Hye Park, "Parenthood, Professorship, and Librarianship: Are They Mutually Exclusive?" Journal of Academic Librarianship 34, no. 3 (2008): 209.

29. Ibid.

30. Ward and Wolf-Wendel, Academic Motherhood, 52.

31. Graves et al., "Parenthood, Professorship, and Librarianship," 202.

32. Shalau Gillium, "The True Benefit of Faculty Status for Academic Reference Librarians," Reference Librarian 51, no. 4 (2010): 321.

33. "Association of College and Research Libraries Joint Statement on Faculty Status of College and University Librarians," American Library Association, available online at www.ala.org/acrl/ standards/jointstatementfaculty [accessed 22 September 2014].

34. Quinn Galbraith, "Librarian Faculty Status and Tenure: A Quick Glance at Where We Stand," (unpublished manuscript, Dec. 3, 2010).

35. Ward and Wolf-Wendel, Academic Motherhood, 57.

36. June, "Fear of Stigma Lingers"; Bahn, "When Grad School Eats Up Your 'Good Years.'”

37. Graves et al., "Parenthood, Professorship, and Librarianship," 202.

38. Andrew E. Clark, "Job Satisfaction and Gender: Why Are Women So Happy at Work?" Labour Economics 4, no. 4 (1997): 365.

39. Ibid.

40. Ward and Wolf-Wendel, Academic Motherhood, 50.

41. Ibid., 51.

42. Simpson, "Masculinity at Work," 356.

43. Shannon K. McCoy, Ellen E. Newell, and Susan K. Gardner, "Seeking Balance: The Importance of Environmental Conditions in Men and Women Faculty's Well-Being," Innovative Higher Education 38, no.4 (2013): 319.

44. Ibid.

45. E. Jeffrey Hill, "Work-Family Facilitation: Expanding Theoretical Understanding Through Qualitative Exploration," Advances in Developing Human Resources 9, no. 4 (2007): 523. 
46. Spencer, "Taking Care of Yourself," 15-17.

47. Spires, "The Busy Librarian," 104-105.

48. Sheesley, "Burnout and the Academic Teaching Librarian," 449.

49. Gabriel, "Dealing with Stress," 472; Sheesley, "Burnout and the Academic Teaching Librarian," 450; Harwell, "Burnout Strategies for Librarians," 387.

50. E. Jeffrey Hill et al., "Defining and Conceptualizing Workplace Flexibility," Community, Work \& Family 11, no. 2 (2008): 152.

51. Hill et al., "Finding an Extra Day a Week," 54. 